\title{
TASA DE INCIDENCIA DEL ACCIDENTE CEREBROVASCULAR EN EL PERÚ
}

\author{
Antonio Bernabé-Ortiz@1,2,a, Rodrigo M. Carrillo-Larco@2,3,b \\ 1 CRONICAS Centro de Excelencia en Enfermedades Crónicas, Universidad Peruana Cayetano Heredia, Lima, Perú. \\ 2 Universidad Científica del Sur, Lima, Perú. \\ ${ }^{3}$ Department of Epidemiology and Biostatistics, School of Public Health, Imperial College London, Londres, Reino Unido. \\ a Médico cirujano, magíster en Salud Pública, doctor en Epidemiología; ${ }^{\text {}}$ médico cirujano.
}

\section{RESUMEN}

Objetivo: Determinar la tasa de incidencia del accidente cerebro vascular (ACV) total y por subtipos en el Perú entre 2017 y 2018. Materiales y métodos: Análisis de datos de morbilidad hospitalaria obtenidos de la Superintendencia Nacional de Salud (datos abiertos). A partir de los códigos CIE-10 se estudiaron la hemorragia subaracnoidea (I60), la hemorragia intracerebral atraumática (I61), el infarto cerebral (I63) y el ACV no especificado (I64). Se estimó la tasa de incidencia cruda y estandarizada por edad (comparando contra la población de la Organización Mundial de la Salud) y se usó como denominador el número de personas según año, edad y sexo de acuerdo con proyecciones nacionales. Resultados: En 2017, se registró un total de 10570 casos de ACV, mientras que en 2018 hubo 12 835. Los eventos isquémicos fueron más frecuentes en ambos años. Independientemente del subtipo de ACV y del año, los varones fueron más afectados que las mujeres. En la población de 35 años a más, se evidenció un aumento en la incidencia cruda de ACV total entre 2017 y 2018, de 80,9 a 96,7 por 100000 personas-año. La incidencia estandarizada mostró la misma tendencia, pero en mayor magnitud: de 93,9 a 109,8 por 100000 personas-año. El ACV isquémico fue el que más aumentó, con una tasa estandarizada en mayores de 35 años de 35,2 en 2017 y de 46,3 en 2018 por 100000 personas-año. Conclusiones: La incidencia de ACV es elevada en el Perú, con predominio de casos isquémicos y afectando desproporcionalmente a los varones. Estos resultados sugieren la necesidad de tener un sistema de vigilancia para cuantificar la incidencia de estos casos y entender sus determinantes.

Palabras clave: Accidente Cerebrovascular; Accidente Cerebrovascular Isquémico; Accidente Cerebrovascular Hemorrágico; Hemorragia Subaracnoidea; Epidemiología; Incidencia; Perú (fuente: DeCS BIREME).

\section{INCIDENCE RATE OF STROKE IN PERU}

\begin{abstract}
Objective: To determine the incidence of stroke, overall and by sub-types, in Peru between 2017 and 2018. Materials and Methods: Analysis of hospital morbidity data obtained from SUSALUD (open data). Using the ICD-10 codes, the following were studied: subarachnoid hemorrhage (I60), atraumatic intracerebral hemorrhage (I61), cerebral infarction (I63), and unspecified stroke (I64). The crude and age-standardized incidence was calculated according to the population of the World Health Organization and using the national projected population number of people according to year, age and sex as the denominator. Results: In 2017, a total of 10,570 stroke cases were recorded, whereas, in 2018, there were 12,835 cases. Ischemic events were more frequent in both years. Regardless of stroke subtype and year, men were more affected than women. In the $35+$ year-old population, an increase in the crude incidence of total stroke was observed between 2017 and 2018, from 80.9 to 96.7 per 100,000 person-years. The age-standardized incidence showed the same trend, but in a greater magnitude: from 93.9 to 109.8 per 100,000 person-years. Ischemic stroke was the one that increased the most, with an age-standardized rate in people aged 35+ years of 35.2 in 2017 and 46.3 per 100,000 person-years in 2018. Conclusions: The incidence of stroke is high in Peru. Ischemic cases are the most frequent and they disproportionately affect men. Our results suggest the need for a surveillance system to robustly quantify the incidence of these cases and understand their determinants.
\end{abstract}

Keywords: Stroke; Ischemic Stroke; Hemorrhagic Stroke; Subarachnoid Hemorrhage; Epidemiology, Incidence; Peru (source: MeSH NLM).

\section{INTRODUCCIÓN}

Recibido: $03 / 04 / 2021$

En todo el mundo, los eventos cardiovasculares son la principal causa de muerte ${ }^{(1)}$. Entre ellos, En línea: 30/09/2021 
y mortalidad (2). Para 2016, se estimó que alrededor de 5,5 millones de muertes fueron secundarias al $\mathrm{ACV}^{(3)}$, y que la tasa de incidencia de ACV en países de bajos y medianos ingresos excede a la incidencia en países de altos ingresos ${ }^{(4)}$.

Aunque la incidencia de ACV viene disminuyendo desde 1990, más del 80\% de los casos de los ACV ocurren en países de escasos recursos económicos ${ }^{(5)}$, con estimados de incidencia en 117 por 100000 personas-año, que varía entre 73 y 165 por 100000 personas-año ${ }^{(4)}$. Sin embargo, existe limitada vigilancia de esta condición en estos países, tal es el caso del Perú. Por ejemplo, se desconoce cuál es la incidencia poblacional de ACV, en forma total y por subtipo de ACV, ya que la mayoría de la información proviene de modelos matemáticos con datos internacionales aplicados a nuestro contexto.

En la literatura se encuentran diversas formas de determinar la incidencia de ACV en la población. Así, algunos autores han determinado la incidencia de ACV usando estudios longitudinales de tipo cohorte ${ }^{(6)}$, otros lo han hecho estableciendo sistemas de registro de ACV en ciertas regiones de determinados países ${ }^{(7)}$, o usando el autorreporte de sintomatología neurológica compatible con ACV (incluyendo el uso de imágenes o evaluación especializada) ocurrida en el año previo a la evaluación ${ }^{(8)}$. Sin embargo, son pocos los estudios que han utilizado registros nacionales de ACV para determinar no solo la incidencia de ACV, sino también la incidencia de los subtipos de dichos eventos.

Como resultado, el presente trabajo trata de estimar la tasa de incidencia de ACV, en total y de acuerdo al subtipo de ACV, en forma cruda y estandarizada por edad, usando registros nacionales de hospitalización de los años 2017 y 2018.

\section{MATERIALES Y MÉTODOS}

\section{Diseño y lugar de estudio}

Se realizó un estudio de datos secundarios, basado en los registros de ACV ocurridos en 2017 y 2018 en el Perú. La información usada en el presente análisis proviene de los registros de hospitalización disponibles en la página web de la Superintendencia Nacional de Salud (SUSALUD) ${ }^{(9)}$. Dicha información contiene datos de todo el sistema nacional de salud: Ministerio de Salud, Direcciones Regionales de Salud, Seguro Social (ESSALUD), Sanidad de las Fuerzas Armadas y Policiales, y sector privado.

Las bases de datos cuentan con información sobre el año y mes de diagnóstico del ACV, la región, provincia y distrito donde se registró dicho evento, así como la edad y sexo del caso hospitalizado. Adicionalmente, se cuenta con el respectivo diagnóstico codificado según la Clasificación Internacional de Enfermedades versión 10 (CIE-10) y el número de

\section{MENSAJES CLAVE}

Motivación para hacer el estudio: La evidencia sobre la prevención y manejo de los accidentes cerebrovasculares (ACV) en el Perú proviene principalmente de estimados internacionales o de algunos estudios hospitalarios locales.

Principales hallazgos: Los resultados de este estudio muestran una aproximación a la tasa de incidencia de ACV a nivel poblacional en el Perú, siendo mayor a más edad y en varones. De acuerdo con el subtipo de ACV, aquellos de índole isquémica fueron los que tuvieron mayor incidencia en los dos años de estudio, mientras que existe aún una gran proporción de ACV que no pueden ser especificados apropiadamente.

Implicancias: La incidencia de ACV en el Perú es elevada. Urge un sistema de vigilancia para cuantificar la incidencia de estos casos y entender sus determinantes.

total de atendidos por mes, lo que permitió estimar el número de casos hospitalizados con las condiciones de interés. Sin embargo, la base de datos no cuenta con detalles de atención en emergencia o de mortalidad.

\section{Criterios de selección}

Este análisis contiene todos los casos registrados con diagnóstico de ACV definido según el CIE-10, independientemente del sexo y de la edad a la que ocurrió el evento.

\section{Definición de variables}

Se definió el ACV según el diagnóstico de cualquier evento de hospitalización (nuevo episodio o episodio recurrente) basado en el CIE-10. De esta forma, se utilizó I60 para definir hemorragia subaracnoidea (HSA); I61 para hemorragia intracerebral atraumática compatible con ACV hemorrágico; I63 para infarto cerebral compatible con ACV isquémico; e I64 para ACV no especificado. Adicionalmente, se agruparon todos los casos antes definidos para estimar el número de eventos compatibles con ACV y estimar la incidencia total. No se usaron los otros códigos, porque no estaban asociados a daño cerebral propio del ACV agudo (I65, I66 o I67) o estaban relacionados a secuelas de enfermedad cerebrovascular (I68 o I69).

Otras variables de interés fueron el sexo (varón, mujer); la edad, evaluada en grupos ( $<35,35-44,45-54,55-64, y \geq 65)$, aunque el mayor interés estuvo en los eventos de 35 a más años; sector de salud donde se registró el caso (MINSA, ESSALUD, Sanidad, y privado/otros); y el año de reporte $(2017,2018)$.

\section{Análisis estadístico}

Se utilizó el programa STATA versión 16 para Windows (StataCorp, College Station, TX, EUA) y Excel 2019 (Micro- 
soft, WA, EUA) para el análisis. Inicialmente, se tabularon los eventos de interés reportados por año, subtipo de ACV y sector de salud donde se registraron. Asimismo, se describió brevemente la población con diagnóstico de ACV.

Luego, se estimó la tasa de incidencia de ACV sobre la base del número de casos reportados por año por cada 100000 habitantes. El denominador se definió según la población en riesgo cada año, sobre la base de las proyecciones de población total, por años calendario, sexo y edad del Instituto Nacional de Estadística e Informática (INEI) publicada en el $2009^{(10)}$. De esta forma, se pudo estimar la incidencia total, por sexo, y grupos de edad, como el número de casos de ACV dividido por la población en riesgo en cada grupo de interés y año específico. La decisión de usar las proyecciones se basó en que el censo nacional del 2017 solo serviría para ese año y se usaría proyecciones para el 2018, lo cual podría afectar drásticamente los resultados.

Debido a los pocos casos de ACV en las personas menores de 35 años, en relación con la población en riesgo, y a fin de comparar con otros estudios internacionales, se puso énfasis en las personas de 35 años a más. Se estimó también la tasa de incidencia de ACV estandarizada por edad de acuerdo a la distribución de la población de la Organización Mundial de la Salud (OMS) usando el método directo ${ }^{(11)}$. De acuerdo con esto, se reportó la incidencia de ACV por sexo y grupo de edad y sus respectivos intervalos de confianza al 95\% (IC 95\%).

Finalmente, los mismos pasos anteriormente descritos se usaron para estimar la tasa de incidencia de ACV de acuerdo con el subtipo (HSA, hemorrágico, isquémico, y no especificado).

\section{Criterios éticos}

Para el presente análisis se usaron datos secundarios disponibles en la página web de SUSALUD. La información se encuentra agrupada en forma mensual por centro de atención y sin identificadores personales. En consecuencia, este trabajo no fue sometido a revisión ética.

\section{RESULTADOS}

\section{Descripción de los casos con ACV}

Se evaluó un total de 2147747 (994 537 en 2017 y 1153210 en 2018) registros de hospitalización. De los cuales, 2119465 (98,7\%) tenían el respectivo diagnóstico CIE-10 (99,5\% en 2017 y $97,9 \%$ en 2018) y se usaron en el presente análisis.

Para 2017, se registró un total de 10570 casos de ACV (46,6\% en mujeres), mientras que en 2018 fue de 12835 casos de ACV (44,9\% en mujeres). La mayoría de los casos de ACV provenían del Ministerio de Salud tanto para 2017 como para 2018 (Tabla 1).

Con respecto al subtipo de ACV, para 2017, 12,0\% de los eventos fueron HSA; $16,7 \%$, hemorragia cerebral; $35,7 \%$, eventos isquémicos; y $35,6 \%$, ACV no especificados. Para 2018, 10,0\% fueron HSA; 17,7\%, ACV hemorrágicos; 40,4\%, $\mathrm{ACV}$ isquémicos; $\mathrm{y} 31,9 \%$, ACV no especificados.

\section{Incidencia de ACV total y por sexo}

Para 2017, la tasa de incidencia cruda de ACV fue 33,2 (IC 95\%: 32,6-33,8) por cada 100000 personas-año, la que aumentó a 39,9 (IC 95\%: 39,2-40,6) en 2018. Cuando los estimados se estandarizaron de acuerdo con la población descrita por la OMS, estos fueron 38,1 (IC 95\%: 37,5-38,8) y 44,9 (IC 95\%: 44,2-45,7) para 2017 y 2018, respectivamente.

Cuando solo se analizó a aquellos de 35 años a más, la tasa de incidencia cruda de ACV incrementó de 80,9 (IC 95\%: 79,3-82,5) en 2017 a 96,7 (IC 95\%: 95,0-98,1) por 100000 personas-año en 2018. Dichos valores estandarizados fueron 93,9 (IC 95\%: 92,2-95,7) en 2017 y 109,8 (IC 95\%: 108,0111,7) en 2018.

Para todos los grupos de edad y años de estudio, la tasa de incidencia de ACV siempre fue mayor en varones que en mujeres (Tabla 2).

Tabla 1. Número de casos de accidente cerebrovascular según tipo y procedencia.

\begin{tabular}{|c|c|c|c|c|}
\hline Año/subsector & $\begin{array}{c}\text { Hemorragia } \\
\text { subaracnoidea }\end{array}$ & $\begin{array}{c}\text { ACV } \\
\text { hemorrágico }\end{array}$ & $\begin{array}{c}\mathrm{ACV} \\
\text { isquémico }\end{array}$ & $\begin{array}{c}\text { ACV } \\
\text { no especificado }\end{array}$ \\
\hline \multicolumn{5}{|l|}{2017} \\
\hline MINSA & 638 & 926 & 1113 & 1919 \\
\hline ESSALUD & 449 & 567 & 2073 & 1270 \\
\hline Sanidad de las Fuerzas Armadas y Policiales & 17 & 63 & 143 & 58 \\
\hline Privado u otros & 161 & 214 & 440 & 519 \\
\hline \multicolumn{5}{|l|}{2018} \\
\hline MINSA & 631 & 1340 & 2104 & 2173 \\
\hline ESSALUD & 450 & 627 & 2361 & 1190 \\
\hline Sanidad de las Fuerzas Armadas y Policiales & 16 & 52 & 139 & 40 \\
\hline Privado u otros & 186 & 248 & 586 & 692 \\
\hline
\end{tabular}

ACV: accidente cerebrovascular; MINSA: Ministerio de Salud; ESSALUD: Seguro Social de Salud 
Tabla 2. Tasa de incidencia de accidente cerebrovascular (por 100000 personas-año) por edad y sexo (2017-2018).

\begin{tabular}{|c|c|c|c|c|c|c|}
\hline \multirow{2}{*}{ Año/grupo etario } & \multicolumn{2}{|r|}{ Varones } & \multicolumn{2}{|r|}{ Mujeres } & \multicolumn{2}{|r|}{ Total } \\
\hline & Casos & TI (IC 95\%) & Casos & TI (IC 95\%) & Casos & TI (IC 95\%) \\
\hline \multicolumn{7}{|l|}{2017} \\
\hline \multicolumn{7}{|l|}{ Incidencia cruda } \\
\hline$<35$ & 363 & $3,66(3,28-4,03)$ & 285 & $2,96(2,62-3,30)$ & 648 & $3,31(3,06-3,57)$ \\
\hline $35-44$ & 244 & $11,11(9,71-12,51)$ & 234 & $10,73(9,36-12,11)$ & 478 & $10,93(9,95-11,90)$ \\
\hline $45-54$ & 549 & $32,80(30,05-35,54)$ & 482 & $28,49(25,94-31,03)$ & 1031 & $30,63(28,76-32,50)$ \\
\hline $55-64$ & 990 & $87,18(81,75-92,60)$ & 714 & $59,93(55,53-64,32)$ & 1704 & $73,22(69,75-76,70)$ \\
\hline$\geq 65$ & 3495 & $347,89(336,38-359,40)$ & 3214 & $269,82(260,50-279,13)$ & 6709 & $305,54(298,24-312,84)$ \\
\hline Total & 5641 & $35,39(34,47-36,31)$ & 4929 & $31,03(30,16-31,89)$ & 10570 & $33,21(32,58-33,84)$ \\
\hline$\geq 35$ & & $87,83(85,46-90,20)$ & & $74,25(72,12-76,39)$ & & $80,90(79,31-82,50)$ \\
\hline \multicolumn{7}{|c|}{ Incidencia estandarizada } \\
\hline Total & & $43,24(42,24-44,27)$ & & $33,61(32,71-34,51)$ & & $38,13(37,45-38,81)$ \\
\hline$\geq 35$ & & $109,04(106,41-111,68)$ & & $81,00(78,77-83,23)$ & & $93,93(92,22-95,65)$ \\
\hline \multicolumn{7}{|l|}{2018} \\
\hline \multicolumn{7}{|l|}{ Incidencia cruda } \\
\hline$<35$ & 360 & $3,62(3,25-3,99)$ & 321 & $3,33(2,96-3,69)$ & 681 & $3,48(3,21-3,74)$ \\
\hline $35-44$ & 296 & $13,29(11,77-14,80)$ & 255 & $11,53(10,11-12,94)$ & 551 & $12,41(11,37-13,45)$ \\
\hline $45-54$ & 687 & $40,12(37,12-43,12)$ & 539 & $31,16(28,53-33,79)$ & 1226 & $35,62(33,63-37,61)$ \\
\hline $55-64$ & 1168 & $99,39(93,69-105,08)$ & 810 & $65,72(61,20-70,25)$ & 1978 & $82,15(78,53-85,77)$ \\
\hline$\geq 65$ & 4555 & $437,29(424,62-449,96)$ & 3844 & $310,99(301,17-320,81)$ & 8399 & $368,75(360,88-376,62)$ \\
\hline Total & 7066 & $43,87(42,85-44,90)$ & 5769 & $35,93(35,00-36,85)$ & 12835 & $39,91(39,22-40,60)$ \\
\hline$\geq 35$ & & $108,92(106,31-111,52)$ & & $84,99(82,73-87,24)$ & & $96,71(94,99-98,43)$ \\
\hline \multicolumn{7}{|c|}{ Incidencia estandarizada } \\
\hline Total & & $52,72(51,60-53,84)$ & & $38,11(37,12-39,11)$ & & $44,94(44,21-45,67)$ \\
\hline$\geq 35$ & & $132,39(129,51-135,26)$ & & $83,63(81,39-85,87)$ & & $109,82(107,99-111,65)$ \\
\hline
\end{tabular}

TI: tasa de incidencia. IC 95\%: intervalo de confianza al 95\%

\section{Incidencia de ACV de acuerdo con el subtipo}

Para 2018, la tasa de incidencia global de HSA fue 4,0 (IC 95\%: 3,8-4,2); la de ACV hemorrágico, 7,1 (IC 95\%: 6,8-7,3); y la de ACV isquémico, 16,1 (IC 95\%: 15,7-16,6) por 100000 personas-año. De otro lado, la tasa de incidencia estandarizada de HSA fue 4,3 (IC 95\%: 4,1-4,5); de ACV hemorrágico, 7,8 (IC 95\%: 7,5-8,1); y de ACV isquémico, 18,4 (IC 95\%: 17,918,8). Mientras que los estimados para HSA fueron similares a los de 2017, la incidencia de ACV hemorrágico y de ACV isquémico fueron mayores en 2018 que en 2017 (Tabla 3). De otro lado, la incidencia de ACV no especificado incrementó de 11,8 (IC 95\%: 11,5-12,2) en 2017 a 12,7 (IC 95\%: 12,3-13,1) por 100000 personas-año en 2018 (Material suplementario).

\section{DISCUSIÓN}

En los dos años evaluados, los resultados del presente estudio detallan la tasa de incidencia de ACV en el Perú, siendo mayor especialmente en aquellos de mayor edad y en varones. Aunque dos años consecutivos pueden ser relativamente poco tiempo para determinar tendencias, también se evidencia un incremento en el número de casos de ACV con predominio de las causas hemorrágicas e isquémicas. De acuerdo con el subtipo de ACV, aquellos de índole isquémica tuvieron mayor incidencia en los dos años de estudio, mientras que existe aún una gran proporción de ACV que no se pueden especificar apropiadamente.

Muchos de los trabajos previos que han estimado la prevalencia de ACV han sido transversales ${ }^{(12,13)}$. Sin embargo, según el Estudio Mundial de Carga de Enfermedad (GBD, por sus siglas en inglés), la incidencia cruda de ACV en el Perú para todas las edades fluctúa entre 73 y 74 por 100000 personas-año para 2017 y 2018, respectivamente ${ }^{(14)}$. Nuestros hallazgos subestiman la incidencia de ACV frente a los resultados mostrados por el GBD, debido al uso de registros identificados mediante códigos CIE-10, mientras que los 
Tabla 3. Tasa de incidencia de accidente cerebrovascular por subtipos (por 100000 personas-año) por edad y tipo (2017 - 2018).

\begin{tabular}{|c|c|c|c|c|c|c|}
\hline \multirow{2}{*}{ Año/grupo etario } & \multicolumn{2}{|c|}{ Hemorragia subaracnoidea } & \multicolumn{2}{|c|}{ ACV hemorrágico } & \multicolumn{2}{|c|}{$\mathrm{ACV}$ isquémico } \\
\hline & Casos & TI (IC 95\%) & Casos & TI (IC 95\%) & Casos & TI (IC 95\%) \\
\hline \multicolumn{7}{|l|}{2017} \\
\hline \multicolumn{7}{|l|}{ Incidencia cruda } \\
\hline$<35$ & 217 & $1,11(9,62-1,26)$ & 213 & $1,09(0,94-1,24)$ & 77 & $0,39(0,31-0,48)$ \\
\hline $35-44$ & 128 & $2,93(2,42-3,43)$ & 99 & $2,26(1,82-2,71)$ & 112 & $2,56(2,09-3,03)$ \\
\hline $45-54$ & 236 & $7,01(6,12-7,91)$ & 209 & $6,21(5,37-7,05)$ & 285 & $8,47(7,48-9,45)$ \\
\hline $55-64$ & 251 & $10,79(9,45-12,12)$ & 306 & $13,15(11,68-14,62)$ & 620 & $26,64(24,55-28,74)$ \\
\hline$\geq 65$ & 433 & $19,72(17,86-21,58)$ & 943 & $42,95(40,21-45,69)$ & 2675 & $121,82(117,21-126,44)$ \\
\hline Total & 1265 & $3,97(3,76-4,19)$ & 1770 & $5,56(5,30-5,82)$ & 3769 & $11,84(11,46-12,22)$ \\
\hline$\geq 35$ & & $8,55(8,03-9,06)$ & & $12,70(12,07-13,33)$ & & $30,10(29,13-31,08)$ \\
\hline \multicolumn{7}{|c|}{ Incidencia estandarizada } \\
\hline Total & & $4,36(4,13-4,59)$ & & $6,28(6,00-6,55)$ & & $13,78(13,37-14,19)$ \\
\hline$\geq 35$ & & $9,65(9,10-10,20)$ & & $14,64(13,96-15,31)$ & & $35,16(34,11-36,21)$ \\
\hline \multicolumn{7}{|l|}{2018} \\
\hline \multicolumn{7}{|l|}{ Incidencia cruda } \\
\hline$<35$ & 236 & $1,20(1,05-1,36)$ & 246 & $1,26(1,10-1,41)$ & 89 & $0,45(0,36-0,55)$ \\
\hline $35-44$ & 125 & $2,82(2,32-3,31)$ & 123 & $2,77(2,28-3,26)$ & 170 & $3,83(3,25-4,40)$ \\
\hline $45-54$ & 227 & $6,59(5,74-7,45)$ & 265 & $7,70(6,77-8,63)$ & 408 & $11,85(10,70-13,00)$ \\
\hline $55-64$ & 241 & $10,01(8,75-11,27)$ & 390 & $16,20(14,59-17,81)$ & 706 & $29,32(27,16-31,49)$ \\
\hline$\geq 65$ & 454 & $19,93(18,10-21,77)$ & 1243 & $54,57(51,54-57,61)$ & 3817 & $167,58(162,27-172,89)$ \\
\hline Total & 1283 & $3,99(3,77-4,21)$ & 2267 & $7,05(6,76-7,34)$ & 5190 & $16,14(15,70-16,58)$ \\
\hline$\geq 35$ & & $8,33(7,83-8,84)$ & & $16,08(15,38-16,78)$ & & $40,59(39,48-41,70)$ \\
\hline \multicolumn{7}{|c|}{ Incidencia estandarizada } \\
\hline Total & & $4,31(4,08-4,54)$ & & $7,82(7,52-8,13)$ & & $18,36(17,89-18,83)$ \\
\hline$\geq 35$ & & $9,23(8,70-9,76)$ & & $18,14(17,40-18,89)$ & & $46,31(45,12-47,50)$ \\
\hline
\end{tabular}

ACV: accidente cerebrovascular; TI: tasa de incidencia; IC 95\%: intervalo de confianza al 95\%

estimados del GBD son obtenidos a través de modelos matemáticos, por la falta de datos en el Perú.

Se han realizado otros estudios similares en contextos parecidos, como en países cercanos al Perú. Por ejemplo, el estudio de incidencia de ACV en Joinville ${ }^{(15)}$, en Brasil, ha reportado valores de incidencia estandarizada de 90,9 por 100000 habitantes para 2013, pero que vienen descendiendo desde 1995, donde la incidencia estandarizada era de 143,7 por 100000 personas-año. De otro lado, la incidencia estandarizada de ACV en Durango, México, se ha estimado en 270,7 por 100000 personas-año mediante un sistema de vigilancia establecido en la ciudad ${ }^{(16)}$. En Nuble (Chile), la incidencia de un primer evento de ACV se ha estimado en 121,7 por 100000 personas-año, los autores recolectaron la información de hospitalización, atención ambulatoria y registros de mortalidad ${ }^{(17)}$. En Tandil, Argentina, se ha estimado la incidencia estandarizada de ACV en 88,1 por 100000 personas-año, mediante una búsqueda activa de casos en diversos registros ${ }^{(18)}$. Finalmente, en Uruguay, se estimó la incidencia de ACV en 181,4 casos por cada 100000 personas- año mediante un sistema de registro y captación de casos en los servicios de urgencias de los centros asistenciales de la ciudad de Rivera ${ }^{(19)}$.

Los hallazgos del presente estudio, aunque se encuentran por debajo de todos estos estimados, son la primera aproximación para estimar la incidencia de ACV en la población peruana, menos estudios aún han estimado la incidencia de ACV de acuerdo con el subtipo. Cabral et al. ${ }^{(20)}$, usando los datos del estudio de Joinville, determinaron que la incidencia estandarizada de $\mathrm{ACV}$ isquémico (86,0 por cada 100000 personas-año) fue mucho más alta que la incidencia de hemorragia cerebral (12,9 por 100000 personasaño) y que la de HSA (7,0 por 100000 personas-año). De igual manera, Bahit et al., en Argentina, ${ }^{(18)}$ estimaron que la incidencia estandarizada de ACV isquémico (65,2 por 100000 personas-año) fue mucho más alta que la de ACV hemorrágico (15,2 por 100 000 personas-año) habitantes y HSA (5,2 por 100000 personasaño). Así, los resultados del presente estudio muestran la misma tendencia, donde los ACV de subtipo isquémico son los más frecuentes y presentan mayor incidencia a nivel poblacional. 
Estos hallazgos reafirman la necesidad de mejorar el sistema de registro de casos de ACV en el Perú, lo cual podría lograrse tomando la definición normalizada de ACV de la OMS, implementando instrumentos de vigilancia paso a paso de ACV (registro) e integrando la información de las distintas fuentes. De ser el caso, se podrían obtener estimados más fiables que ayuden a determinar la verdadera carga de esta condición en nuestro país. Aunque existe una aparente mejora en el registro de ACV, esto debe sostenerse apropiadamente en el tiempo para cerrar la brecha epidemiológica.

De otro lado, la mayor incidencia de ACV en las personas de 35 a más años implica la necesidad de un tratamiento apropiado de los factores de riesgo asociados al desarrollo de estos eventos cerebrovasculares, entre ellos, la hipertensión, la diabetes mellitus tipo 2 y la hipercolesterolemia, así como otros de causa embólica, como la fibrilación auricular.

En el Perú se ha estimado que la prevalencia de hipertensión arterial en la población se viene incrementando, con una reducción en la proporción de hipertensos que conocen su diagnóstico, así como en aquellos que están debidamente controlados ${ }^{(21)}$. Asimismo, la fracción atribuible de ACV debido a hipertensión ha sido estimada en casi $50 \%{ }^{(22)}$, lo que resalta la necesidad de un diagnóstico y tratamiento apropiado de los casos de hipertensión. Con la diabetes ocurre algo similar, con un incremento en la prevalencia a través de los años ${ }^{(23)}$ y una gran proporción de personas que no conocen su diagnóstico y, por lo tanto, no se encuentran debidamente controladas ${ }^{(24)}$. Sin embargo, los datos sobre hipercolesterolemia o fibrilación auricular son casi inexistentes en nuestro contexto.

Que un gran porcentaje de los casos de ACV se clasifiquen como ACV no especificado puede indicar las carencias del sistema de salud para realizar un diagnóstico apropiado de estos eventos, en especial, debido a la ausencia de imágenes (ej., tomografía computarizada), las cuales se realizan generalmente en establecimientos de tercer nivel de atención ${ }^{(25)}$. Así, aunque se puede llegar al diagnóstico clínico de ACV, no se puede determinar su subtipo, lo que resalta la urgente necesidad de garantizar este tipo de herramientas diagnósticas.

Este es uno de los primeros intentos de estimar la incidencia de ACV por sexo, grupo de edad y subtipo en la población del Perú. Los estimados son conservadores y muestran consistencia en los dos años evaluados. Sin embargo, este estudio tiene algunas limitaciones que merecen ser resaltadas.

\section{REFERENCIAS BIBLIOGRÁFICAS}

1. Roth GA, Johnson C, Abajobir A, Abd-Allah F, Abera SF, Abyu G, et al. Global, Regional, and National Burden of Cardiovascular Diseases for 10 Causes, 1990 to 2015. J Am Coll Cardiol. 2017;70(1):1-25. doi: 10.1016/j.jacc.2017.04.052.

2. Feigin VL, Forouzanfar MH, Krishnamurthi R, Mensah GA, Connor $\mathrm{M}$, Bennett DA, et al. Global and regional burden of stroke during
Primero, el presente trabajo se basa en los registros existentes del sistema de salud del Perú y, por ende, puede existir subregistro, lo que podría subestimar los valores de incidencia. Este problema puede deberse a errores en el registro, una mala asignación del tipo de caso al usar el CIE-10, o a que la información consignada se basa en casos que accedieron al sistema de salud (hospitalización). Es esperable que una proporción importante de casos de ACV puede no haber sido registrado, con la subsecuente subestimación de la incidencia real de ACV ${ }^{(25)}$. Esto suele ser más patente en el ámbito rural ${ }^{(26)}$. Así, el aumento de un año a otro puede ser un verdadero aumento en el número de casos de $\mathrm{ACV}$, pero también deberse a una mejora paulatina en el registro. Sin embargo, nuestros resultados son conservadores y están dentro de los valores esperados para la región, como se ha reportado previamente ${ }^{(4)}$.

Segundo, la proporción de casos de ACV de causa no especificada es demasiado grande en comparación con otros trabajos donde ese tipo de ACV no existe o es demasiado pequeño. Si bien la proporción de estos casos no especificados se reduce de un año a otro, lo que puede implicar una mejora en el acceso a la salud y en el registro de la información, esto puede tener un gran impacto en la incidencia de los otros subtipos de ACV.

Tercero, no hay cómo definir apropiadamente si los casos registrados fueron un primer evento o un ACV recurrente. Asimismo, podría existir una pequeña fracción de personas con historia de dos o más ACV en un mismo año.

Finalmente, se determinó el número de personas en riesgo usando proyecciones para nuestra población. Su efecto debería ser negligible, ya que no ha habido cambios bruscos en el tamaño de la población en los años de estudio.

En conclusión, los resultados de este estudio demuestran una alta tasa de incidencia de ACV en la población del Perú, siendo mayor en las personas de mayor edad y en varones. También se evidencia que los casos isquémicos son los más preponderantes. Existe la necesidad de tener un sistema apropiado de registro de casos de ACV para poder estimar apropiadamente la carga de esta condición en nuestro país.

Contribuciones de los autores: $\mathrm{ABO}$ y RMCL concibieron, diseñaron y redactaron el artículo, y aprobaron su versión final. $\mathrm{ABO}$ realizó los análisis estadísticos requeridos, y RMCL revisó críticamente sus contenidos.

\section{Conflicto de interés: Ninguno}

Financiamiento: RMCL ha sido financiado por una beca de Wellcome Trust (214185/Z/18/Z).

Material suplementario: Disponible en la versión electrónica de la RPMESP.
1990-2010: findings from the Global Burden of Disease Study 2010. Lancet. 2014;383(9913):245-54. doi: 10.1016/s0140-6736(13)61953-4. 3. Global Burden of Disease 2016 Stroke Collaborators. Global, regional, and national burden of stroke, 1990-2016: a systematic analysis for the Global Burden of Disease Study 2016. Lancet Neurol. 2019;18(5):43958. doi: 10.1016/s474-4422(19)30034-1. 
4. Feigin VL, Lawes CM, Bennett DA, Barker-Collo SL, Parag V. Worldwide stroke incidence and early case fatality reported in 56 population-based studies: a systematic review. Lancet Neurol. 2009;8(4):35569. doi: 10.1016/s474-4422(09)70025-0.

5. Strong K, Mathers C, Bonita R. Preventing stroke: saving lives around the world. Lancet Neurol. 2007;6(2):182-7. doi: 10.1016/s474-4422(07)70031-5.

6. Meza E, Eng CW, Sáenz JL, Gilsanz P, Glymour MM, Torres JM. Elevated Depressive Symptoms and the Risk of Stroke among the Mexican Older Population. J Am Geriatr Soc. 2020;68(11):2579-86. doi: 10.1111 /jgs.16718.

7. Minelli C, Cabral NL, Ujikawa LT, Borsetti Neto FA, Langhi Chiozzini EM, Dos Reis GC, et al. Trends in the Incidence and Mortality of Stroke in Matão, Brazil: The Matão Preventing Stroke (MAPS) Study. Neuroepidemiology. 2020;54(1):75-82. doi: 10.1159/000503005.

8. Del Brutto OH, DEl Brutto VJ, Zambrano M, Lama J. [Cerebrovascular disease in Atahualpa: Prevalence and Incidence]. Rev Ecuat Neurol. 2017;26(2):158-63.

9. Superintendencia Nacional de Salud. Consulta D2 - Consolidado de Morbilidad en Hospitalización. Lima, Perú: SUSALUD; 2021 [visitado 24 enero de 2021]; Disponible en: http://datos.susalud.gob.pe/dataset/ consulta-d2-consolidado-de-morbilidad-en-hospitalizacion.

10. Instituto Nacional de Estadística e Informática. Perú: Estimaciones y Proyecciones de Población Total, por años calendario y edades simples, 1950 (Boletín Especial Nº17). Lima, Perú: INEI; 2009.

11. Ahmad OB, Boschi-Pinto C, Lopez AD, Murray CJL, Lozano R, Inoue M. Age standardization of rates: a new WHO standard. Geneva, Switzerland: WHO; 2001.

12. Jaillard AS, Hommel M, Mazetti P. Prevalence of stroke at high altitude $(3380 \mathrm{~m})$ in Cuzco, a town of Peru. A population-based study. Stroke. 1995;26(4):562-8. doi: 10.1161/01.str.26.4.562.

13. Sousa RM, Ferri CP, Acosta D, Guerra M, Huang Y, Jacob K, et al. The contribution of chronic diseases to the prevalence of dependence among older people in Latin America, China and India: a 10/66 Dementia Research Group population-based survey. BMC Geriatr. 2010;10:53. doi: 10.1186/471-2318-10-53.

14. Institute for Health Metrics and Evaluation. Global Health Data Exchange (GHDx). Seattle, WA, US: IHME; 2019 [visitado 31 de marzo de 2021]; Disponible en: http://ghdx.healthdata.org/gbd-results-tool.

15. Cabral NL, Cougo-Pinto PT, Magalhaes PS, Longo AL, Moro CH, Amaral CH, et al. Trends of Stroke Incidence from 1995 to 2013 in Joinville, Brazil. Neuroepidemiology. 2016;46(4):273-81. doi: 10.1159/000445060.

16. Cantu-Brito C, Majersik JJ, Sánchez BN, Ruano A, Becerra-Mendoza $\mathrm{D}$, Wing JJ, et al. Door-to-door capture of incident and prevalent stroke cases in Durango, Mexico: the Brain Attack Surveillance in Durango Study. Stroke. 2011;42(3):601-6. doi: 10.1161/strokeaha.110.592592.

17. Lavados PM, Hoffmeister L, Moraga AM, Vejar A, Vidal C, Gajardo $\mathrm{C}$, et al. Incidence, risk factors, prognosis, and health-related quality of life after stroke in a low-resource community in Chile (NANDU): a prospective population-based study. Lancet Glob Health. 2021;9(3):e340-e51. doi: 10.1016/s2214-109x(20)30470-8.

18. Bahit MC, Coppola ML, Riccio PM, Cipriano LE, Roth GA, Lopes RD, et al. First-Ever Stroke and Transient Ischemic Attack Incidence and 30-Day Case-Fatality Rates in a Population-Based Study in Argentina. Stroke. 2016;47(6):1640-2. doi: 10.161/strokeaha.116.013637.

19. Hochmann B, Coelho J, Segura J, Galli M, Ketzoian C, Pebet M. [The incidence of cerebrovascular accidents in the town of Rivera, Uruguay]. Rev Neurol 2006;43(2):78-83.

20. Cabral NL, Gonçalves AR, Longo AL, Moro CH, Costa G, Amaral $\mathrm{CH}$, et al. Incidence of stroke subtypes, prognosis and prevalence of risk factors in Joinville, Brazil: a 2 year community based study. J Neurol Neurosurg Psychiatry. 2009;80(7):755-61. doi: 10.1136/ jnnp.2009.172098.

21. Villarreal-Zegarra D, Carrillo-Larco RM, Bernabe-Ortiz A. Shortterm trends in the prevalence, awareness, treatment, and control of arterial hypertension in Peru. J Hum Hypertens. 2021;35(5):463-71. doi: 10.1038/s41371-020-0361-1.

22. Kjeldsen SE, Narkiewicz K, Burnier M, Oparil S. The INTERSTROKE Study: hypertension is by far the most important modifiable risk factor for stroke. Blood Press. 2017;26(3):131-2. doi: 10.1080/08037051.2017.1292456.

23. Carrillo-Larco RM, Bernabé-Ortiz A. [Type 2 diabetes mellitus in peru: a systematic review of prevalence and incidence in the general population]. Rev Peru Med Exp Salud Publica. 2019;36(1):26-36. doi: 10.17843/rpmesp.2019.361.4027.

24. Bernabé-Ortiz A, Carrillo-Larco RM, Gilman RH, Checkley W, Smeeth L, Miranda JJ. Contribution of modifiable risk factors for hypertension and type- 2 diabetes in Peruvian resource-limited settings. J Epidemiol Community Health. 2016;70(1):49-55. doi: 10.1136/jech-2015-205988.

25. Miranda JJ, Moscoso MG, Yan LL, Diez-Canseco F, Málaga G, Garcia $\mathrm{HH}$, et al. Addressing post-stroke care in rural areas with Peru as a case study. Placing emphasis on evidence-based pragmatism. J Neurol Sci. 2017;375:309-15. doi: 10.1016/j.jns.2017.02.027.

26. Hammond G, Luke AA, Elson L, Towfighi A, Joynt Maddox KE. Urban-Rural Inequities in Acute Stroke Care and In-Hospital Mortality. Stroke. 2020;51(7):2131-8. doi: 10.1161/strokeaha.120.029318. 